\title{
Double inhibition of NF-KB and XIAP via RNAi enhances the sensitivity of pancreatic cancer cells to gemcitabine
}

\author{
LI-PING CAO ${ }^{1}$, JIAN-LIN SONG ${ }^{1}$, XIAO-PING YI ${ }^{2}$ and YI-XIONG LI ${ }^{1}$ \\ Departments of ${ }^{1}$ General Surgery and ${ }^{2}$ Radiology, Xiangya Hospital, Central South University, Hunan 410008, P.R. China
}

Received October 9, 2012; Accepted December 28, 2012

DOI: 10.3892/or.2013.2246

\begin{abstract}
The majority of patients with pancreatic cancer are resistant to gemcitabine. One of the mechanisms involved is the anti-apoptotic ability of these cells. The median lethal dose $\left(\mathrm{LD}_{50}\right)$ of gemcitabine for PANC-1 cells was higher than that for Mia PaCa-2 cells and the former had higher nuclear factor- $\kappa \mathrm{B}(\mathrm{NF}-\kappa \mathrm{B})$ and X-linked inhibitor of apoptosis protein (XIAP) levels. NF- $\kappa \mathrm{B}$ contributes to the inhibition of apoptosis by the downregulation of downstream genes, such as XIAP and Bcl-2 and it confers chemoresistance. The two cell lines were infected with NF- $\kappa$ B p65 small interfering RNA (siRNA). p65 protein was effectively downregulated accompanied by the downregulation of XIAP protein. The combination treatment with gemcitabine and p65 siRNA increased the apoptotic rates in both cell lines; however, this was not sufficient. XIAP is involved in apoptosis to a greater extent compated to Bcl-2. XIAP may serve as another factor affecting the sufficiency of chemotherapy. XIAP siRNA was designed to knockdown XIAP. Mia PaCa-2 and PANC-1 cells were co-infected with XIAP siRNA and p65 siRNA. XIAP and p65 proteins were effectively downregulated and the gemcitabine-induced apoptotic rates were significantly increased. These results suggest that XIAP and NF- $\kappa \mathrm{B}$ are two important factors conferring the chemoresistance of pancreatic cancer cells, and that their downregulation via RNAi effectively enhances the chemosensitivity of pancreatic cancer cells to gemcitabine.
\end{abstract}

\section{Introduction}

Pancreatic cancer is the fourth leading cause of cancer-related mortality in Western countries. The medial survival rate for patients with pancreatic cancer is approximately five months and the five-year survival rate is approximately 5\% for all stages of the disease (1). Chemotherapy is the main treatment regimen for pancreatic cancer. Gemcitabine monotherapy is

Correspondence to: Dr Yi-Xiong Li, Department of General Surgery, Xiangya Hospital, Central South University, Changsha, Hunan 410008, P.R. China

E-mail: liyixiong2011@hotmail.com

Key words: nuclear factor- $\kappa \mathrm{B}, \mathrm{X}$-linked inhibitor of apoptosis protein, RNAi, chemoresistance, gemcitabine, pancreatic cancer currently the first-line therapy recommended by the National Comprehensive Cancer Network (NCCN) panel (2). However, the majority of patients are resistant to gemcitabine (3). One of the potential mechanisms involved is insensitivity to gemcitabine-induced apoptosis $(4,5)$. Nuclear factor- $\kappa \mathrm{B}$ $(\mathrm{NF}-\kappa \mathrm{B})$ and $\mathrm{X}$-linked inhibitor of apoptosis protein (XIAP) are two important factors in the apoptotic pathway (6), which render them promising targets in reversing the chemoresistance of pancreatic cancer cells.

Mammalian NF- $\mathrm{NB}$ is a family of ubiquitous transcription factors formed by homo- or heterodimers of five $\mathrm{NF}-\kappa \mathrm{B}$ members: Rel A (p65), c-Rel (Rel), Rel B, NF-кB1 (p50) and NF- $\kappa$ B2 (p52) (7). The heterodimer p65/p50 is the most abundant in many types of cells (8). In resting cells, $N F-\kappa B$ is sequestered in the cytoplasm with inhibitory proteins termed I $\mathrm{Bs}$; upon multiple stimuli, such as cytokines, bacterial pathogens or ionizing radiation, the I $\mathrm{B}$ kinase (IKK) complex phosphorylates the I $\kappa \mathrm{B}$ molecules at conserved serine residues, leading to the ubiquitination and degradation of $\mathrm{I} \kappa \mathrm{B}$ by the $26 \mathrm{~S}$ proteasome. $\mathrm{NF}-\kappa \mathrm{B}$ is subsequently released from $\mathrm{I} \kappa \mathrm{B}$ and translocates to the nucleus to promote the transcription of various target genes (9). NF- $\kappa \mathrm{B}$ most commonly suppresses apoptosis by activating the transcription of anti-apoptotic genes, such as XIAP and Bcl-2 family genes (10).

XIAP belongs to the inhibitor of apoptosis proteins (IAPs) including eight family members defined by the presence of the baculovirus IAP repeat (BIR) domain (11). IAPs function mainly by regulating caspases involved in apoptosis. Of all IAPs, mammalian XIAP is the only one that directly inhibits caspases. During apopotosis, XIAP inhibits caspase-9, -3 and -7 to protect cell against death (12).

It has been reported that several NF- $\kappa \mathrm{B}$ subunits, particularly the p65 subunit, are overexpressed in pancreatic cancer cells and that patients with pancreatic cancer with a high expression of p65 have a poor prognosis $(13,14)$. There are encouraging results which suggest that $\mathrm{NF}-\kappa \mathrm{B}$ is an excellent therapeutic target for pancreatic cancer. Arlt et al (15) demonstrated that the inhibition of NF- $\mathrm{NB}$ activation sensitized pancreatic cancer chemoresistant cells towards gemcitabine treatment. Kong et al (16) demonstrated the inhibition of $\mathrm{NF}-\kappa \mathrm{B}$ protein expression by the transfection of p65 small interfering RNA (siRNA) synergized with gemcitabine to induce apoptosis. However, Pan et al (17) did not completely agree with these conclusions. In their study, they concluded that silencing NF- $\kappa \mathrm{B}$ p65 led to gemcitabine-induced apoptosis only in chemosensitive pancrea- 
tic cancer cells but not in resistant ones. Therefore, the issue that remains is that the inhibition of $\mathrm{NF}-\kappa \mathrm{B}$ alone may not be as effective in increasing the chemosensitivity of pancreatic cancer cells as demonstrated previously.

On the basis of its ability to inhibit caspase activity, XIAP has been described as a chemoresistance factor in ammalian cancer (18). Elevated XIAP expression has been reported in pancreatic cancer patients and has been found to be associated with chemoresistance and decreased patient survival (19). The silencing of XIAP can enhance the chemosensitivity of pancreatic cancer cells $(19,20)$.

As $\mathrm{NF}-\kappa \mathrm{B}$ is a vital transcription factor regulating XIAP and a possible chemoresistant factor, we hypothesized that $\mathrm{NF}-\kappa \mathrm{B}$ in conjunction with XIAP may confer the chemoresistance of pancreatic cancer cells; simultaneously targeting $\mathrm{NF}-\kappa \mathrm{B}$ and XIAP by RNAi may enhance chemosusceptibility to gemcitabine.

\section{Materials and methods}

Cell lines and reagents. The human pancreatic cancer cell lines, Mia PaCa-2 and PANC-1, were stored in liquid nitrogen in the Cell Bank of the State Key Laboratory of Medical Genetics, China. When used, they were taken out and revivified. The cells were routinely cultured in Dulbecco's modified Eagle's medium (DMEM) plus $10 \%$ fetal bovine serum, streptomycin $(100 \mathrm{mg} / \mathrm{ml})$ and penicillin $(100 \mathrm{U} / \mathrm{ml})$ at $37^{\circ} \mathrm{C}$ in a humidified incubator containing $5 \% \mathrm{CO}_{2}$. Gemcitabine (Lilly France, Fegersheim, France) was a gift from Dr Zhidong Wang, Gerontism Hospital of Hunan Province.

Transfection of siRNA targeting $N F-\kappa B$ p 65 subunit or XIAP. p65 siRNA (sense, 5'-CCAUCAACUAUGAUGAGUUdTdT-3 and antisense, 3'-dGdTGGUAGUUGAUACUACUCAA-5') (17) was designed to target the NF- $\mathrm{B}$ p 65 subunit. XIAP siRNA (sense, 5'-GGUGAAGGUGAUAAAGUAA-3' and antisense, 3'-CCACUUCCACUAUUUCAUU-5') (21) was designed to target XIAP. A non-specific siRNA control (sense, 5'-UUCUCC GAACGUGUCACGU-3' and antisense, 3'-AAGAGGCUUGC ACAGUGCA-5') was also designed. The siRNAs were produced by GenePharma Co., Ltd., Shanghai, China. Mia PaCa and PANC-1 cells (40,000 cells from each cell line) were grown in six-well plates until $70 \%$ confluence. The cells were then transfected with p65 siRNA or XIAP siRNA or control siRNA using Lipofectamine ${ }^{\mathrm{TM}} 2000$ (Invitrogen) in medium according to the manufacturer's instructions. After $72 \mathrm{~h}$, the protein was extracted and used for western blot analysis to evaluate the effect of gene silencing.

Electrophoretic mobility shift assay (EMSA). Nuclear extracts were prepared from cells using the NucBuster ${ }^{\mathrm{TM}}$ Protein Exaction kit (Merck, Germany) according to the manufacturer's instructions. Biotin-labeled $\mathrm{NF}-\kappa \mathrm{B}$ oligonucleotides (sequence, 5'-AGTTGAGGGGACTTTCCCAGGC-3' and 3'-TCAACTCCCCTGAAAGGGTCCG-5') were used for gel retardation assay. The obtained nuclear extracts $(3 \mu \mathrm{g}$ protein) were incubated with the biotin-labeled NF- $\kappa \mathrm{B}$ oligonucleotides at room temperature for $20 \mathrm{~min}$ and subjected to electrophoresis and chemiluminescent reaction. Competition was performed by adding specific unlabeled double-stranded oligonucleotide to the reaction mixture in 100-fold molar excess. The gels were dried and visualized with a Cool Imager imaging system (IMGR002).

Western blot analysis. Cells were washed in PBS, pH 7.4, lysed and homogenized in RIPA buffer containing $50 \mathrm{mM}$ Tris- $\mathrm{HCl}$, $\mathrm{pH}$ 7.4, $150 \mathrm{mM} \mathrm{NaCl}, 1 \mathrm{mM}$ EDTA, 1\% NP-40, 0.1\% SDS and $0.5 \%$ sodium deoxycholate supplemented with protease inhibitor cocktail set (Pierce, Rockford, IL, USA). The lysed material was collected and centrifuged at $4{ }^{\circ} \mathrm{C}$ for $10 \mathrm{~min}$ at $9,000 \mathrm{rpm}$. The total protein concentration of the supernatant was measured using the BCA assay kit (Sigma, Inc.). Proteins were run on $10 \%$ polyacrylamide SDS gels and transferred onto nitrocellulose membranes. The blots were blocked for $2 \mathrm{~h}$ at room temperature with $5 \%$ non-fat milk power. The blots were then incubated with primary antibodies and subsequently with secondary antibodies. Equal loading and transfer were confirmed using an anti-GAPDH antibody (Santa Cruz Biotechnology, Inc.). Primary and secondary antibodies used were: i) mouse anti-human XIAP polyclonal antibody (Santa Cruz Biotechnology, Inc.), 1:80,000; secondary antibody $1: 10,000$; ii) rabbit anti-human NF- $\mathrm{NB}$ p65 polyclonal antibody (Abcam, Inc.), 1:40,000; secondary antibody 1:10,000.

Detection of apoptosis. Apoptosis was assayed by Annexin VFITC binding to the externalized phosphatidylserine. Apoptosis was monitored with the assay kit and protocol provided by the supplier (Beyotime Institute of Biotechnology, China). The apoptosis rate was analyzed by fluorescence-activated cell sorting analysis.

Detection of cell growth inhibition rate. Analysis of the cell growth inhibition rate was used to determine the sensitivity of the cell lines to gemcitabine. Mia PaCa-2 and PANC-1 cells were seeded in $100 \mu \mathrm{l}$ medium in 96-well plates and various concentrations of gemcitabine were added to the plates. After incubation for $72 \mathrm{~h}, 10 \mu \mathrm{l}$ water-soluble tetrazolium (WST-8) were added to each well and incubated for $1 \mathrm{~h}$. The $\mathrm{OD}_{450} \mathrm{~nm}$ was measured. The cell growth inhibition rate was calculated using the following formula: percentage cell growth inhibition rate $=100 \%-[($ detected cell OD value - blanket control OD value)/(control cell OD value - blanket control OD value)].

Statistical analysis. All data are expressed as the means \pm SD. Analysis was performed using analysis of variance (ANOVA). A value of $\mathrm{P}<0.05$ was considered to indicate a statistically significant difference.

\section{Results}

Gemcitabine causes greater cell growth inhibition in Mia PaCa-2 compared to PANC-1 cells. Mia PaCa-2 and PANC-1 cells were treated with various concentrations of gemcitabine for $72 \mathrm{~h}$. Cell viability was determined by the CCK-8 assay. The $\mathrm{LD}_{50}$ of gemcitabine was approximately $1 \mu \mathrm{M}$ for Mia PaCa-2 and $>50 \mu \mathrm{M}$ for PANC-1 cells (Fig. 1). Mia PaCa-2 cells showed greater sensitivity to gemcitabine compared to PANC-1 cells. We classified Mia PaCa-2 as gemcitabine-sensitive and PANC-1 as gemcitabine-resistant based on their different $\mathrm{LD}_{50}$ values. 


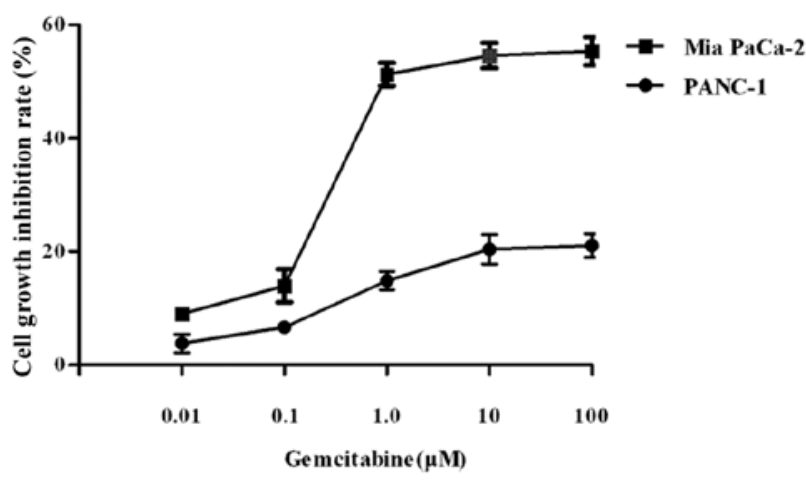

Figure 1. Cell growth inhibition ability of gemcitabine in human pancreatic cancer cells. Mia PaCa-2 and PANC-1 cells were treated with varying concentrations $(0.01-100 \mu \mathrm{M})$ of gemcitabine for $72 \mathrm{~h}$. The number of viabilities was detected by the CCK- 8 assay. Cell growth inhibition rate was calculated by the following formula: percentage cell growth inhibition rate $=100 \%-[$ (detected cell OD value - blanket control OD value)/(control cell OD value - blanket control OD value)].

Mia PaCa-2 cells have a much lower basal level of $N F-\kappa B$ and XIAP compared to PANC-1 cells. Gemcitabine induced an increase in NF- $\kappa B$ and XIAP levels in the Mia PaCa-2 and PANC-1 cell lines. To determine whether the chemoresitance of pancreatic cancer cells correlates with NF-kB and/or XIAP, we detected their basal and induced levels in Mia PaCa-2 and PANC-1 cells. The cells were treated with or without gemcitabine for $6 \mathrm{~h}$. Nuclear extracts were prepared and EMSAs were performed for NF- $\mathrm{KB}$ DNA binding activity. As shown in Fig. 2A, the Mia PaCa-2 and PANC-1 cells showed a high basal level NF- $\kappa \mathrm{B}$ activity, although the Mia PaCa-2 cells showed a relatively lower level $(\mathrm{P}<0.05)$; gemcitabine induced an increase in NF- $\mathrm{KB}$ activity in both cell lines. Homogenates of the treated cells were used to detect XIAP protein expression by western blot analysis. We observed that the Mia PaCa-2 and PANC-1 cells had high levels XIAP protein, although the Mia PaCa-2 cells had a relatively lower level $(\mathrm{P}<0.05)$; gemcitabine induced an increase in XIAP protein expression in both cell lines $(\mathrm{P}<0.05)$ (Fig. 2B).

p65 siRNA effectivelyinhibits NF- $\kappa$ Bactivityaccompanied by the downregulation of XIAP protein. siRNA targeting NF- $\mathrm{KB}$ p65 was used to knockdown p65. Mia PaCa-2 and PANC-1 cells were separately divided into three groups and treated as follows: untreated; control siRNA or p65 siRNA. Subsequently, western blot analysis was conducted to detect NF- $\mathrm{KB}$ p 65 protein expression (Fig. 3A). In Mia PaCa-2 cells, p65 expression was reduced by $70.85 \pm 0.25 \%$ following treatment with $\mathrm{p} 65$ siRNA compared with control siRNA $(\mathrm{P}<0.05)$. There was no difference between the control siRNA and the untreated groups $(\mathrm{P}>0.05)$. p65 expression was reduced by $54.19 \pm 0.67 \%$ following treatment with p65 siRNA compared with control siRNA $(\mathrm{P}<0.05)$ in PANC-1 cells, and no difference was observed between the control siRNA and the untreated groups $(\mathrm{P}>0.05)$. The cells were then treated with control siRNA, gemcitabine, p65 siRNA or gemcitabine + p65 siRNA. EMSAs were performed. As shown in Fig. 3C, p65 siRNA downregulated basal $(\mathrm{P}<0.05$, compared with control) and gemcitabine-induced $(\mathrm{P}<0.05$, compared with gemcitabine) NF- $\mathrm{KB}$ DNA binding activity in the Mia PaCa-2
A

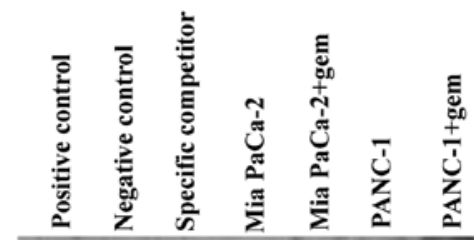

NF-кB

Free probe
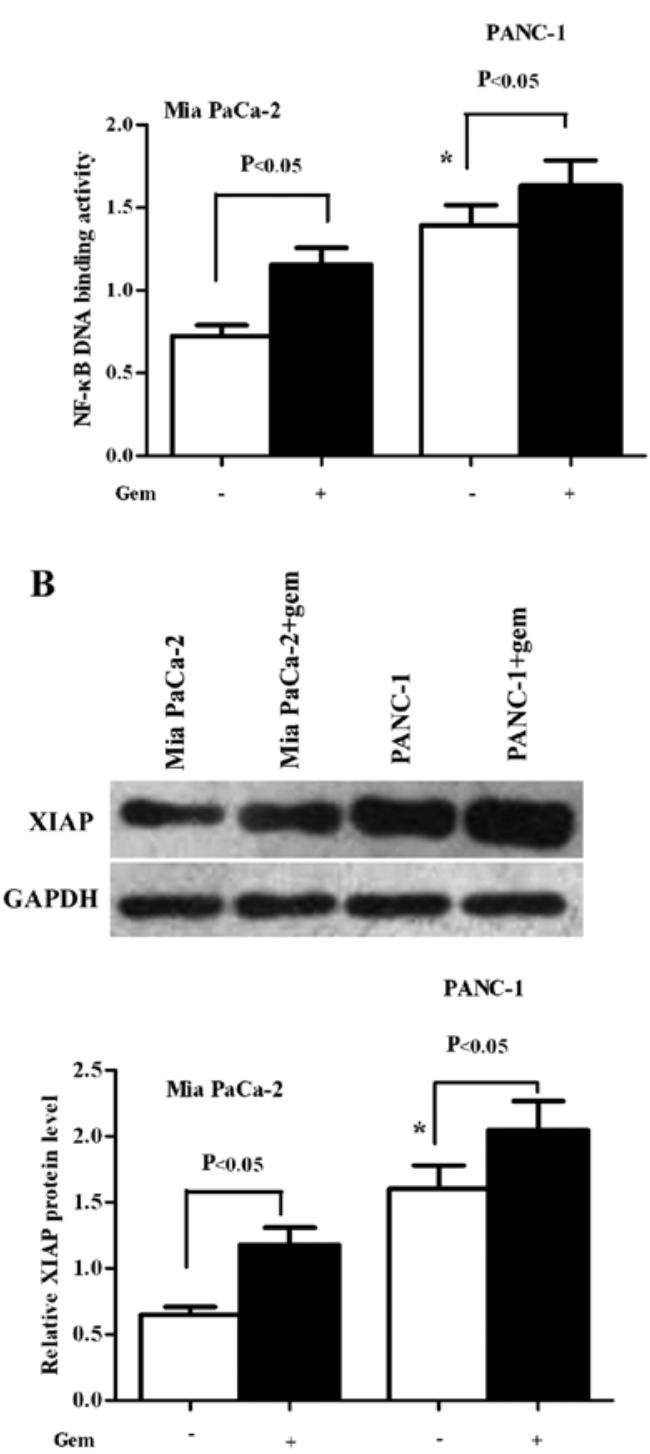

Figure 2. Basal and induced NF- $\kappa$ B activity and XIAP protein level. (A) NF- $\kappa$ B activity. Mia PaCa-2 and PANC-1 cells were treated with or without gemcitabine for $6 \mathrm{~h}$, then nuclear extracts were prepared and EMSA was carried out using a labeled oligonucleotide (Oligo) containing a consensus $\kappa \mathrm{B}$ binding site. Positive control, HeLa nuclear extract and biotin-labeled NF- $\kappa \mathrm{B}$ Oligo; negative control, biotin-labeled NF- $\mathrm{BB}$ Oligo without HeLa nuclear extract; specific competitor, HeLa nuclear extract with biotin-labeled NF- $\kappa \mathrm{B}$ Oligo plus unlabeled NF- $\kappa$ B Oligo. (B) XIAP protein level. Mia PaCa-2 and PANC-1 cells were treated with or without gemcitabine for $6 \mathrm{~h}$. Homogenates were subjected to western blot analysis to detect the expression of XIAP. Columns, means of three experiments; bars, means \pm SD. ${ }^{*} \mathrm{P}<0.05$, versus Mia $\mathrm{PaCa}-2$ without gemciatabine. Gem, gemcitabine.

and PANC-1 cells (Fig. 3C, upper two lanes). To determined whether the inhibition of NF- $\kappa \mathrm{B}$ would affect XIAP expression, western blot analysis was performed. In both cell lines, XIAP 
A

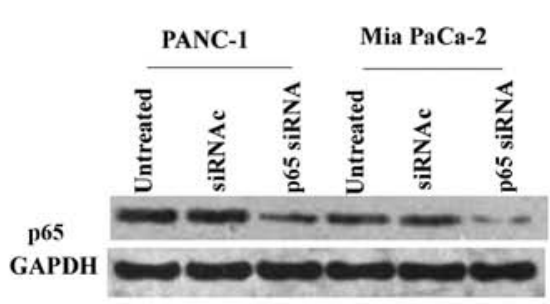

B

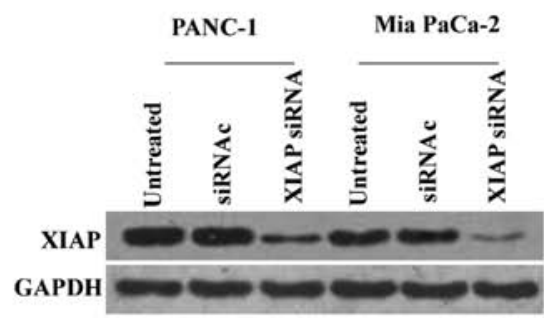

C
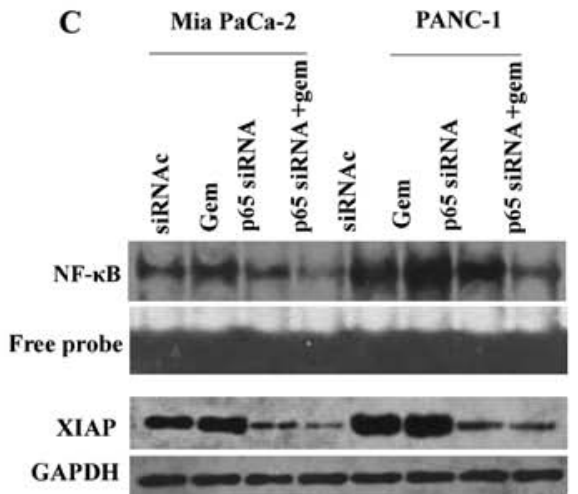

Figure 3. Effectiveness of small interfering RNA (siRNA). Mia PaCa-2 and PANC-1 cells were incubated with antibiotic, control siRNA, p65 siRNA or XIAP siRNA and (A) p65 or (B) XIAP protein levels were detected. The cells were then treated with control siRNA, gemcitabine, p65 siRNA and gemcitabine + p65 siRNA. (C) EMSA was performed to determine NF- $\mathrm{BB}$ DNA binding ability (top two panels); western blot analysis was carried out to detect XIAP protein expression (bottom two panels). Gem, gemcitabine; siRNAc, control siRNA

protein (Fig. 3C, lower two panels) was also downregulated by p65 siRNA compared with control $(\mathrm{P}<0.05)$ or gemcitabine + p65 siRNA compared with gemcitabine $(\mathrm{P}<0.05)$.

p65 siRNA enhance the sensitivity of pancreatic cancer cells to gemcitabine; however, this was not sufficient. Mia PaCa-2 and PANC-1 cells were treated with control siRNA, gemcitabine, p65 siRNA or the combination of gemcitabine and p65 siRNA for $72 \mathrm{~h}$, then stained with Annexin V/PI, and subjected to flow cytometry to measure the rate of apoptosis. As shown in Fig. 4A, gemcitabine alone increased the apoptotic rate compared with the control in both cell lines $(\mathrm{P}<0.05)$; p65 siRNA alone did not alter the apoptotic rate compared with the control siRNA $(\mathrm{P}>0.05)$ in Mia PaCa-2 and PANC-1 cells; the combination of gemcitabine with p65 siRNA increased the apoptotic rate compared with the control siRNA or gemcatabine or p65 siRNA in Mia PaCa-2 and PANC-1 cells $(\mathrm{P}<0.05)$. The representative histograms of the flow cytometry results shown in Fig. $4 \mathrm{~B}$ indicate that the apoptotic rates of the control, gemcitabine, p65 siRNA or the combination of gemcitabine and p65 siRNAtreated groups in Mia PaCa-2 cells were 2.1, 12.3,3.0 and 19.6\%, respectively; in the PANC-1 cells, the rates were 0.8, 4.7, 1.1 and $14.9 \%$, respectively. Although gemcitabine in conjunction with p65 siRNA improved the apoptotic rates of the Mia PaCa-2 and PANC-1 cells, this improvement was not sufficient.

XIAP siRNA effectively downregulates XIAP protein expression. siRNA targeting XIAP was used to knockdown XIAP. Western blot analysis was performed to detect the expression of XIAP protein. As demonstrated in Fig. 3B, XIAP protein expression was reduced by $79.86 \pm 0.37 \%$ in $\mathrm{Mia} \mathrm{PaCa}-2$ and by $65.87 \pm 0.23 \%$ in PANC-1 cells.

XIAP siRNA in conjunction with p65 siRNA downregulate $X I A P$ protein expression and $N F-\kappa B D N A$ binding activity. Mia PaCa-2 and PANC-1 cells were treated with gemcitabine (control), gemcitabine + XIAP siRNA, gemcitabine + p65 siRNA or gemcitabine + XIAP siRNA + p65 siRNA, and subsequently XIAP protein expression and NF- $\kappa \mathrm{B}$ DNA binding activity were detected. In the Mia PaCa-2 and PANC-1 cells, as shown by western blot analysis (Fig. 5, top two panels), gemcitabine
+ XIAP siRNA or gemcitabine + p65 siRNA caused a greater reduction in XIAP protein expression compared with the control $(\mathrm{P}<0.05)$. There was no difference between the groups treated with gemcitabine + XIAP siRNA and gemcitabine + p65 siRNA $(\mathrm{P}>0.05)$. Treatment with XIAP siRNA + p65 siRNA + gemcitabine not only caused a greater reduction in XIAP protein expression compared with the control $(\mathrm{P}<0.05)$, but also compared with XIAP siRNA + gemcitabine or p65 siRNA + gemcitabine treatment. The results of $N F-\kappa B$ DNA binding activity detected by EMSA (Fig. 5, bottom two panels) were the same as those obtained from western blot analysis.

XIAP siRNA and p65 siRNA enhance the chemosensitivity of pancreatic cancer cells to gemcitabine. Mia $\mathrm{PaCa}-2$ and PANC-1 cells were treated as above for $72 \mathrm{~h}$, then stained with Annexin V/PI, and subjected to flow cytometry to measure the rate of apoptosis. As shown in Fig. 6A, in the two types of pancreatic cancer cells, the combination treatment of gemcitabine and XIAP siRNA or gemcitabine and p65 siRNA increased the apoptotic rate compared with the control $(\mathrm{P}<0.05)$. There was no difference between the groups treated with gemcitabine + XIAP siRNA and gemcitabine + p65 siRNA $(\mathrm{P}>0.05)$. The combination treatment of gemcitabine + XIAP siRNA + p65 siRNA increased the apoptotic rate compared with the control $(\mathrm{P}<0.05)$ or gemcitabine + XIAP siRNA $(\mathrm{P}<0.05)$ or gemcitabine + p65 siRNA treatment $(\mathrm{P}<0.05)$. Representative histograms of the flow cytometry results are shown in Fig. 6B. The apoptotic rates of $\mathrm{Mia} \mathrm{PaCa}-2$ cells in the control, gemcitabine + XIAP siRNA, gemcitabine + p65 siRNA, and gemcitabine + XIAP siRNA + p65 siRNAtreated groups were $1.8,16.8,18.5$ and $43.7 \%$, respectively. The corresponding apoptotic rates of the PANC-1 cells were 0.9, $11.1,13.9$ and $39.2 \%$, respectively.

\section{Discussion}

The majority patients with pancreatic cancer show great resistance to chemotherapy. The mechanisms involved remain unknown. One of the potential mechanisms is the insensitivity to drug-induced apoptosis (4). NF- $\mathrm{BB}$ and XIAP play important roles in drug resistance in patients with pancreatic cancer due 
A
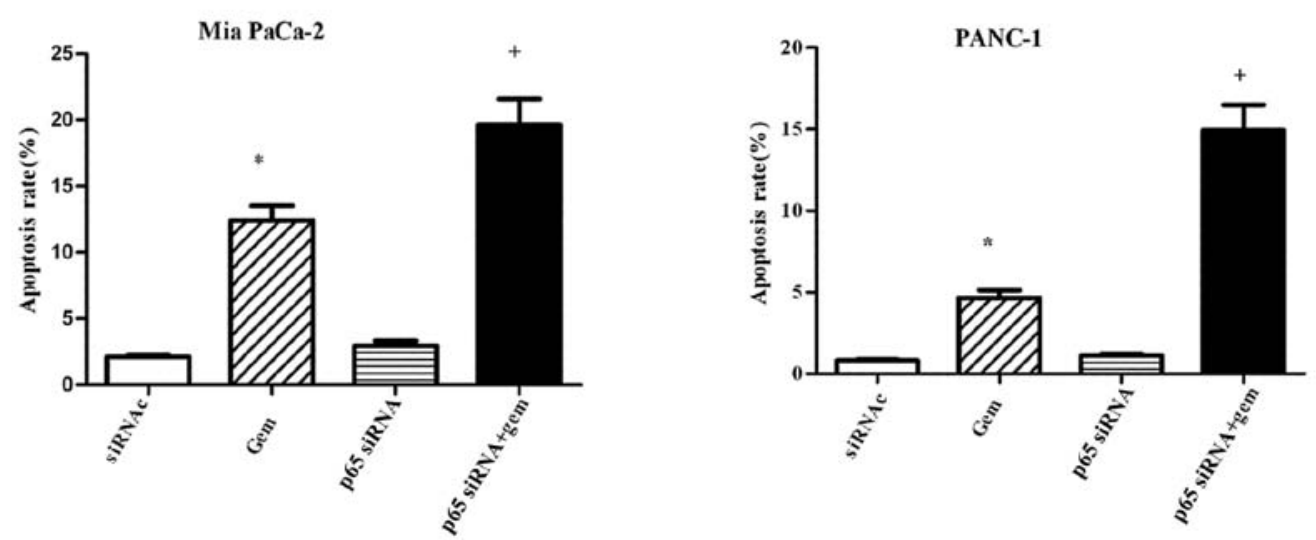

B
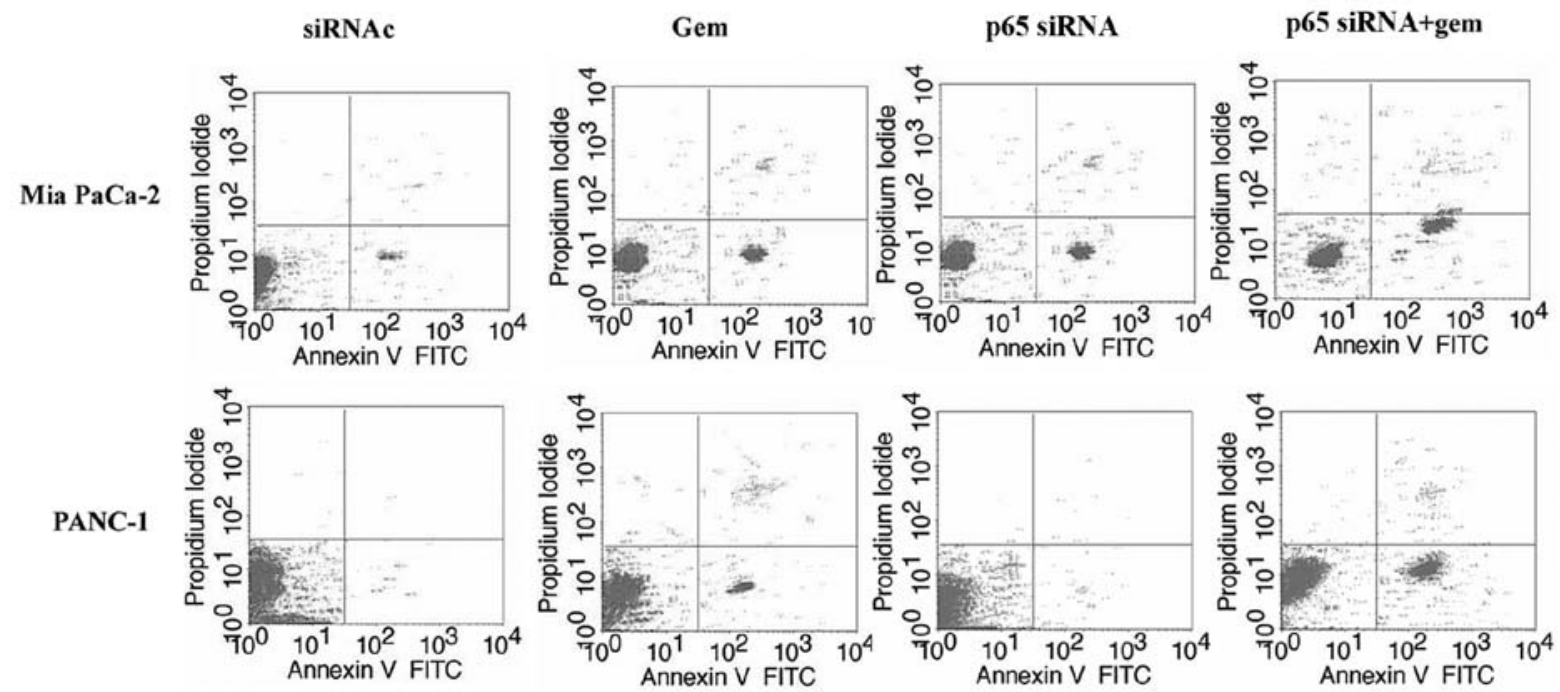

Figure 4. Apoptosis analysis. Mia PaCa-2 and PANC-1 cells were treated with control siRNA, gemcitabine, p65 siRNA and gemcitabine + p65 siRNA. Apoptotic rates were measured by flow cytometry. (A) "Significant difference from control siRNA; +, significant difference from any other three groups. (B) Representative histograms of the flow cytometry of Mia PaCa-2 cells (upper panel) or PANC-1 (lower panel) treated as mentioned above. Gem, gemcitabine; siRNAc, control siRNA.

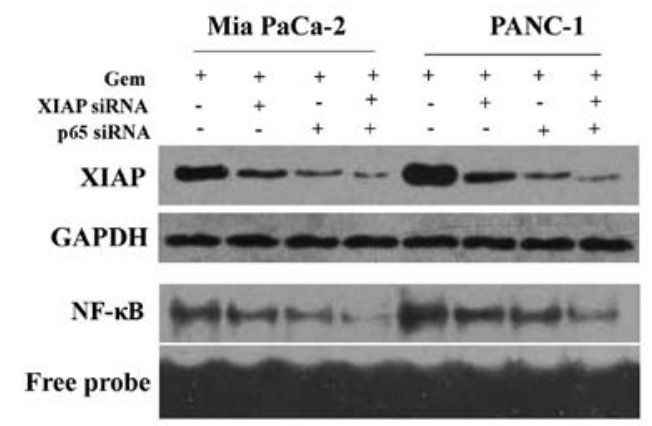

Figure 5. Effectiveness of combined siRNA. Mia PaCa-2 and PANC-1 cells were treated with gem, gem + XIAP siRNA, gem + p65 siRNA, gem + XIAP siRNA + p65 siRNA. XIAP was detected by western blot analysis (top two panels), NF-kB DNA binding ability was detected by EMSA (bottom two panels). Gem, gemcitabine.

to their involvement in the apoptotic pathway $(11,22)$. NF- $\mathrm{kB}$ is constitutively activated in pancreatic cancer specimens as well as in pancreatic cancer cells and contributes to anti-apoptosis (23). The same occurs with XIAP (24). Arlt et al (15) examined six types of pancreatic cancer cells and found that chemoresistant cells had much higher basal levels of NF- $\mathrm{KB}$ than chemosensitive ones and concluded that the basal level of NF- $\mathrm{KB}$ predicted chemoresistance. In the present study, we discovered that PANC-1 cells showed a relatively greater resistance to gemcitabine compared to Mia PaCa-2 cells, as the PANC-1 cells had a higher $\mathrm{LD}_{50}$ of gemcitabine. We classified PANC-1 as gemcitabine-resistant and Mia PaCa-2 as gemcitabine-sensitive. PANC-1 cells had a much higher basal level of XIAP protein and NF- $\kappa B$ DNA binding activity compared to Mia PaCa-2 cells. Therefore, XIAP and NF-kB are two potential factors predicting chemoresistance. To detect the functional association of NF- $\kappa \mathrm{B}$ and XIAP with gemcitabine resistance, we downregulated NF- $\kappa B$ and/or XIAP to determined the effects.

The inhibition of NF- $\mathrm{kB}$ activation by interference with the IKK complex or blocking proteasome activity has been shown to increase apoptosis by suppressing NF- $\kappa \mathrm{B}$ target genes, including XIAP and Bcl-2 (8). IL-32r (25) or some natural products, such as curcumin (26), morin (27) and gossypol (28) have been shown to induce apoptosis or inhibit cancer growth through the suppression of NF- $\mathrm{KB}$ and its downstream genes 
A
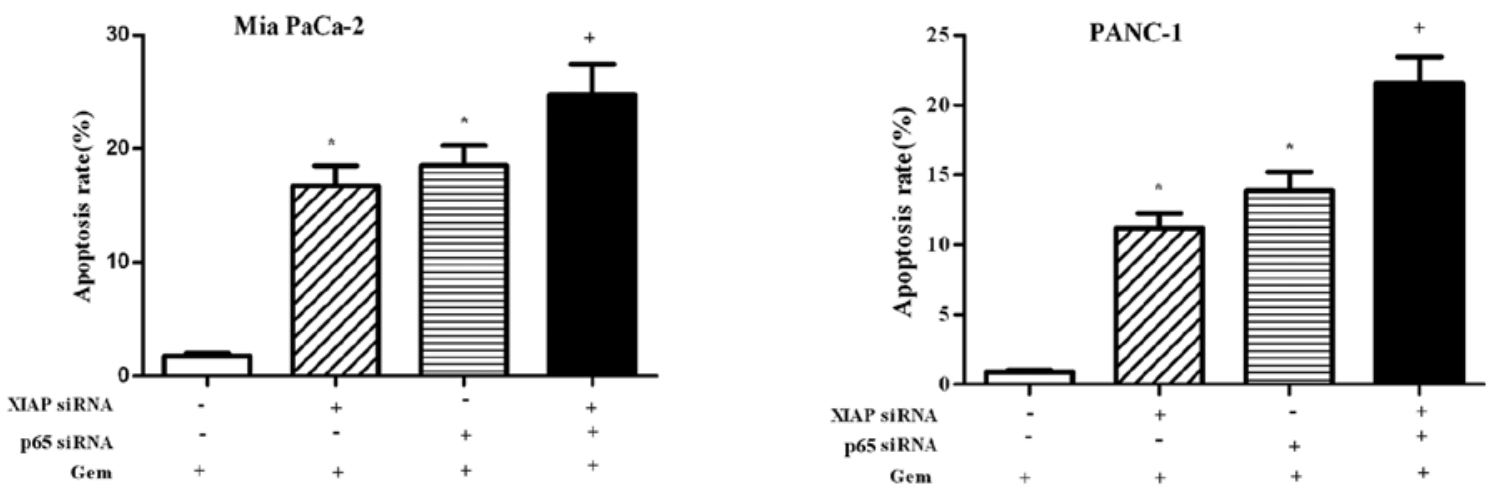

B
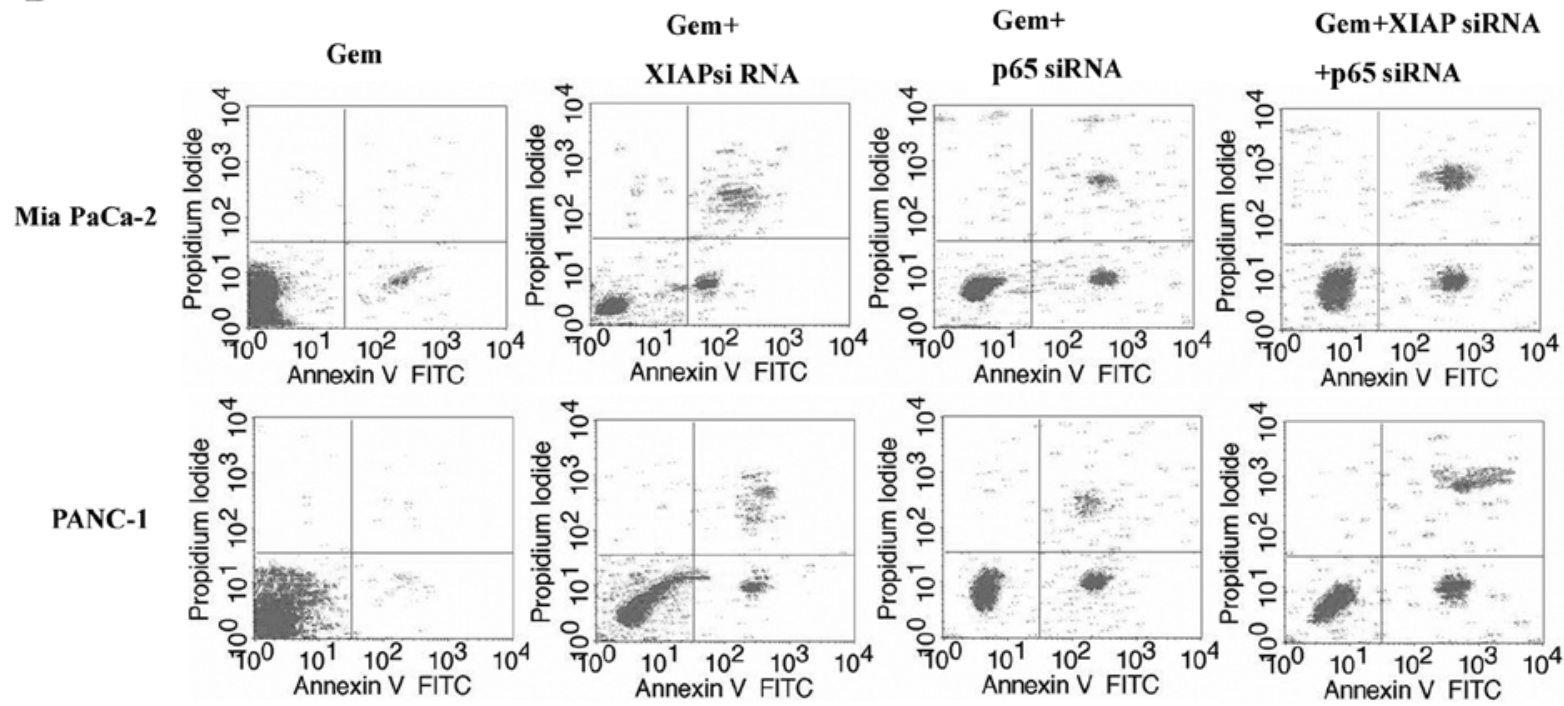

Figure 6. Effect of combined siRNAs in gemcitabine-induced apoptosis. Mia Pa Ca-2 and PANC-1 cells were treated with gem (control), gem + XIAP siRNA, gem + p65 siRNA, gem + XIAP siRNA + p65 siRNA, and apoptotic rates were detected by by flow cytometry. (A) "Significant difference from gem; +, significant difference from any other three groups. (B) Representative histograms of the flow cytometry of Mia PaCa-2 cells (upper panel) or PANC-1 (lower panel) treated as described above. Gem, gemcitabine.

(XIAP and Bcl-2) in many cancer cells. Neither of these methods are specific for the inhibition of NF- $\kappa B$, while RNAi can inhibit the target gene directly (29). In the current study, we designed siRNAs (p65 siRNA and XIAP siRNA) to downregulate the target genes. Gemcitabine induced an increase in NF- $\mathrm{KB}$ DNA binding activity and XIAP protein expression in Mia PaCa-2 and PANC-1 cells, rendering them more chemoresisitant. p65 siRNA effectively downregulated basal and gemcitabine-induced NF- $\mathrm{kB}$ DNA binding activity in the chemoresistant and chemosensitive cells accompanied by the downregulation of XIAP protein expression (which may lead to higher apoptotic rates). As expected, the apoptotic rates of Mia PaCa-2 and PANC-1 cells treated with p65 siRNA combined with gemcitabine were much higher than those of the control, or those treated with gemcitabine or p65 siRNA alone. Therefore, p65 siRNA enhances chemosensitivity in both chemoresistant and chemosensitive cells through the downregulation of XIAP. These results were consistent with those from a previous study (15). However, unlike the results of a previous study (16), the apoptotic rate in our study was not that high. XIAP may be another factor affecting chemoresistance.
$\mathrm{XIAP}$ is a downstream target regulated by NF- $\mathrm{\kappa B}$ and was downregulated when NF- $\kappa \mathrm{B}$ was inhibited by p65 siRNA. However, there remained a possibility that it was not inhibited sufficiently and exerted an influence on the apoptotic rate by the combined treatment of p65 siRNA and gemcitabine. Thus, we designed XIAP siRNA to knockdown XIAP. Bilim et al (30) demonstrated that the inhibition of the XIAP and Bcl-2 axis retrieved the sensitivity of renal cancer cells to apoptosis. We aimed to investigate whether the inhibition of NF- $\kappa \mathrm{B}$ and XIAP would increase the sensitivity of pancreatic cancer cells to gemcitabine. As expected, XIAP siRNA and p65 siRNA downregulated XIAP expression and NF- $\kappa \mathrm{B}$ activity more effectively compared to XIAP siRNA or p65 siRNA alone. The combination treatment of gemcitabine with XIAP siRNA and p65 siRNA increased apoptosis more efficiently than the combination of gemcitabine with XIAP siRNA or gemcitabine with p65 siRNA in both chemoresistant and chemosensitive pancreatic cancer cells. Thus far, there are few studies on the multitargeted therapy of cancer treatment. Kunze et al (31) demonstrated that the targeted inhibition of anti-apoptotic genes (XIAP, Bcl-2 and Bcl-xL) through siRNA combined with cisplatin increased apoptosis in bladder cancer cells. 
Ruckert et al (32) found that the simultaneous gene silencing of Bcl-2, XIAP and survivin without chemotherapy increased the apoptosis of pancreatic cancer cells. In our study, we first demonstrated that the double inhibition of NF- $\mathrm{BB}$ and XIAP through RNAi enhanced the sensitivity of pancreatic cancer cells to gemcitabine. IAPs, particularly XIAP are much more important to cell apoptosis than Bcl-2 (33). XIAP is a better target in cancer therapy. However, Seeger et al (34) demonstrated that XIAP overexpression alone had little effect on chemorisistance to chemotherapeutic agents; only elevated XIAP together with the downregulation of second mitochondria-derived activator of caspases (SMAC), which control XIAP function, conferred chemoresistance in cancer cells. $\mathrm{NF}-\kappa \mathrm{B}$ is an upstream factor regulating XIAP. In our study, we demonstrated that the downregulation of XIAP alone by RNAi was less effective in gemcitabine-induced apoptosis compared to the double inhibition of XIAP and NF- $\kappa \mathrm{B}$. These data show that $\mathrm{NF}-\kappa \mathrm{B}$ and XIAP together confer chemoresistance and that the inhibition of XIAP together with the inhibition of its upstream regulating factor, $N F-\kappa B$, reverse the insensitivity of pancreatic cancer cells to gemcitabine. This method may be used as a novel strategy for the treatment of pancreatic cancer.

In conclusion, XIAP and $\mathrm{NF}-\kappa \mathrm{B}$ are two important anti-apoptotic factors in pancreatic cancer cells. They are overexpressed in either chemoresistant or chemosensitive cells and can predict chemoresistance. The double inhibition of XIAP and NF- $\kappa$ B via RNAi can enhance the chemosensitivity of pancreatic cancer cells to gemcitabine.

\section{Acknowledgements}

This study was supported by a grant from the National Natural Science Foundation of China (no. 30872492) and a grant from the PhD Innovation Program of Hunan Province (no. CX2011B064).

\section{References}

1. Siegel R, Naishadham D and Jemal A: Cancer statistics, 2012. CA Cancer J Clin 62: 10-29, 2012.

2. Tempero MA, Arnoletti JP, Behrman SW, et al: Pancreatic Adenocarcinoma, version 2.2012: featured updates to the NCCN Guidelines. J Natl Compr Cancer Netw 10: 703-713, 2012.

3. Cunningham D, Chau I, Stocken DD, et al: Phase III randomized comparison of gemcitabine versus gemcitabine plus capecitabine in patients with advanced pancreatic cancer. J Clin Oncol 27: 5513-5518, 2009.

4. Gottesman MM: Mechanisms of cancer drug resistance. Annu Rev Med 53: 615-627, 2002.

5. Raguz S and Yague E: Resistance to chemotherapy: new treatments and novel insights into an old problem. Br J Cancer 99: 387-391, 2008.

6. Arlt A, Muerkoster SS and Schafer H: Targeting apoptosis pathways in pancreatic cancer. Cancer Lett: November 13, 2010. doi: 10.1016/j.canlet.2010.10.015

7. Wan $F$ and Lenardo MJ: The nuclear signaling of NF-kappaB: current knowledge, new insights, and future perspectives. Cell Res 20: 24-33, 2010

8. Sebens S, Arlt A and Schafer H: NF-kappaB as a molecular target in the therapy of pancreatic carcinoma. Recent Results Cancer Res 177: 151-164, 2008.

9. Kanarek N, London N, Schueler-Furman O and Ben-Neriah Y: Ubiquitination and degradation of the inhibitors of NF-kappaB. Cold Spring Harb Perspect Biol 2: a000166, 2010.

10. Biswas DK, Martin KJ, McAlister C, et al: Apoptosis caused by chemotherapeutic inhibition of nuclear factor-kappaB activation. Cancer Res 63: 290-295, 2003.
11. Gyrd-Hansen M and Meier P: IAPs: from caspase inhibitors to modulators of NF-kappaB, inflammation and cancer. Nat Rev Cancer 10: 561-574, 2010.

12. Eckelman BP, Salvesen GS and Scott FL: Human inhibitor of apoptosis proteins: why XIAP is the black sheep of the family. EMBO Rep 7: 988-994, 2006.

13. Chandler NM, Canete JJ and Callery MP: Increased expression of NF-kappa B subunits in human pancreatic cancer cells. J Surg Res 118: 9-14, 2004.

14. Weichert W, Boehm M, Gekeler V, et al: High expression of RelA/p65 is associated with activation of nuclear factor-kappaBdependent signaling in pancreatic cancer and marks a patient population with poor prognosis. Br J Cancer 97: 523-530, 2007.

15. Arlt A, Gehrz A, Muerkoster S, et al: Role of NF-kappaB and Akt/ $\mathrm{PI} 3 \mathrm{~K}$ in the resistance of pancreatic carcinoma cell lines against gemcitabine-induced cell death. Oncogene 22: 3243-3251, 2003.

16. Kong R, Sun B, Jiang H, et al: Downregulation of nuclear factorkappaB p65 subunit by small interfering RNA synergizes with gemcitabine to inhibit the growth of pancreatic cancer. Cancer Lett 291: 90-98, 2010.

17. Pan $X$, Arumugam T, Yamamoto $T$, et al: Nuclear factor-kappaB p65/relA silencing induces apoptosis and increases gemcitabine effectiveness in a subset of pancreatic cancer cells. Clin Cancer Res 14: 8143-8151, 2008.

18. Kashkar H: X-linked inhibitor of apoptosis: a chemoresistance factor or a hollow promise. Clin Cancer Res 16: 4496-4502, 2010.

19. Shrikhande SV, Kleeff J, Kayed H, et al: Silencing of X-linked inhibitor of apoptosis (XIAP) decreases gemcitabine resistance of pancreatic cancer cells. Anticancer Res 26: 3265-3273, 2006.

20. Lopes RB, Gangeswaran R, McNeish IA, Wang Y and Lemoine NR: Expression of the IAP protein family is dysregulated in pancreatic cancer cells and is important for resistance to chemotherapy. Int J Cancer 120: 2344-2352, 2007.

21. Li Y, Jian Z, Xia K, et al: XIAP is related to the chemoresistance and inhibited its expression by RNA interference sensitize pancreatic carcinoma cells to chemotherapeutics. Pancreas 32: 288-296, 2006.

22. Fan Y, Dutta J, Gupta N, Fan G and Gelinas C: Regulation of programmed cell death by NF-kappaB and its role in tumorigenesis and therapy. Adv Exp Med Biol 615: 223-250, 2008.

23. Liptay S, Weber CK, Ludwig L, Wagner M, Adler G and Schmid RM: Mitogenic and antiapoptotic role of constitutive NF-kappaB/Rel activity in pancreatic cancer. Int J Cancer 105: 735-746, 2003.

24. Trauzold A, Schmiedel S, Roder C, et al: Multiple and synergistic deregulations of apoptosis-controlling genes in pancreatic carcinoma cells. Br J Cancer 89: 1714-1721, 2003.

25. Oh JH, Cho MC, Kim JH, et al: IL-32 $\gamma$ inhibits cancer cell growth through inactivation of NF- $\kappa$ B and STAT3 signals. Oncogene 30: 3345-3359, 2011.

26. Aggarwal S, Ichikawa H, Takada Y, Sandur SK, Shishodia S and Aggarwal BB: Curcumin (diferuloylmethane) down-regulates expression of cell proliferation and antiapoptotic and metastatic gene products through suppression of IkappaBalpha kinase and Akt activation. Mol Pharmacol 69: 195-206, 2006.

27. Manna SK, Aggarwal RS, Sethi G, Aggarwal BB and Ramesh GT: Morin (3,5,7,2',4'-Pentahydroxyflavone) abolishes nuclear factor-kappaB activation induced by various carcinogens and inflammatory stimuli, leading to suppression of nuclear factor-kappaB-regulated gene expression and up-regulation of apoptosis. Clin Cancer Res 13: 2290-2297, 2007.

28. Moon DO, Kim MO, Lee JD and Kim GY: Gossypol suppresses NF-kappaB activity and NF-kappaB-related gene expression in human leukemia U937 cells. Cancer Lett 264: 192-200, 2008.

29. Hannon GJ: RNA interference. Nature 418: 244-251, 2002.

30. Bilim V, Yuuki K, Itoi T, et al: Double inhibition of XIAP and $\mathrm{Bcl}-2$ axis is beneficial for retrieving sensitivity of renal cell cancer to apoptosis. Br J Cancer 98: 941-949, 2008.

31. Kunze D, Wuttig D, Fuessel S, et al: Multitarget siRNA inhibition of antiapoptotic genes (XIAP, BCL2, BCL-X(L)) in bladder cancer cells. Anticancer Res 28: 2259-2263, 2008.

32. Ruckert F, Samm N, Lehner AK, Saeger HD, Grutzmann R and Pilarsky C: Simultaneous gene silencing of Bcl-2, XIAP and Survivin re-sensitizes pancreatic cancer cells towards apoptosis. BMC Cancer 10: 379, 2010.

33. Liston P, Fong WG and Korneluk RG: The inhibitors of apoptosis: there is more to life than Bcl2. Oncogene 22: 8568-8580, 2003.

34. Seeger JM, Brinkmann K, Yazdanpanah B, et al: Elevated XIAP expression alone does not confer chemoresistance. Br J Cancer 102: 1717-1723, 2010. 